\title{
Research Paper: Effect of Lower Leg Cold Immersion on Dynamic Balance of Athletes and Nonathletes
}

\author{
Ruhollah Salehi ${ }^{1}$, Mostafa Zarei ${ }^{2}$, Mohammad Hossein Alizadeh ${ }^{1}$, Norodin Karimi ${ }^{3}$
}

1. Department of Sport Medicine and Health, Faculty of Physical Education \& Sport Sciences, University of Tehran, Tehran, Iran.

2. Department of Sport and Exercise Physiology, Faculty of Physical Education \& Sport Sciences, Shahid Beheshti University, Tehran, Iran.

3. Department of Physiotherapy, University of Social Welfare and Rehabilitation Sciences, Tehran, Iran.

Citation: Salehi R, Zarei M, Alizadeh MH, Karimi N. [Effect of Lower Leg Cold Immersion on Dynamic Balance of Athlete and Nonathlete (Persian)]. Journal of Rehabilitation. 2016; 17(2):148-157. http://dx.crossref.org/ 10.21859/jrehab-1702148

http://dx.crossref.org/ 10.21859/jrehab-1702148

Received: 13 Mar. 2016 Accepted: 30 May 2016

Keywords: Cryotherapy, Ankle, Foot, Balance

\section{A B S T R ACT}

Objective In recent years, cold water immersion (CWI) following heavy training sessions and matches is commonly used for reducing recovery time. Some studies have investigated the effect of CWI on the performance and physical fitness of athletes. However, there have been no investigations on the effect of cold application on dynamic balance. Therefore, the purpose of this study was to determine the effect of 8-minute cooling of the foot and ankle by immersion in ice on the dynamic balance of athletes and nonathletes.

Materials \& Methods A total of 58 Tehran university students (29 athletes and 29 nonathletes; mean [SD]age: $22.2[2$ ]y, mean [SD] weight: $69.2[2] \mathrm{kg}$, and mean [SD] height: $175.2[2] \mathrm{cm}$ ) consented to participate in the study. They were divided into four groups. The anteroposterior and lateral Indices of dynamic balance were assessed by the Biodex Stability System . In order to familiarize the participants with the Biodex system and prepare them for the evaluation of dynamic balance, each participant was asked to practice on Biodex for one minute. All the subjects were required to immerse their entire feet and ankles in cold water $\left(1^{\circ} \mathrm{C}\right)$ for 8 minutes. The water level was approximately $5 \mathrm{~cm}$ above the lateral malleolus. After the cold application, the dynamic balance of the participants was reassessed. An ANOVA at $\mathrm{P}<0.05$ was used to analyze data.

Results It was found that there was no significant difference in the overall stability before ( $M=2.94$, $S D=1.26)$ and after cryotherapy $(M=2.66, S D=1.40)$. There is no $(t(57)=1.29, P=0.202)$. A significant difference was observed in anterior-posterior stability index before $(M=2.27, S D=1.06)$ and after cryotherapy $(M=2.08, S D=1.19),(t(57)=0.98, P=0.33)$. The lateral stability index after cryotherapy $(M=1.78$, $\mathrm{SD}=0.88)$ showed a significant increase compared to before applying the cold therapy $(\mathrm{M}=2.05$, $\mathrm{SD}=0.84)(\mathrm{t}(57)=2.10, \mathrm{P}=0.03)$.

The results of ANOVA with repeated measures showed that the effect of athlete or nonathletes is not significant on the overall stability index and anterior-posterior $(P=0.48)$ and lateral stability index $(P=0.18)$ before and after cold application.

Conclusion The results of this study suggest that cryotherapy through immersion of foot and ankle does not have a negative effect on the overall and anteroposterior indices of dynamic balance of athletes and nonathletes following an 8-min ice water immersion. It seems that the immersion process affected only the surface receptors of the skin and did not affect the deeper joint receptors that have a key role in balance.

\section{* Corresponding Author:}

\section{Mostafa Zarei, PhD}

Address: Department of Sport and Exercise Physiology, Faculty of Physical Education \& Sport Sciences, Shahid Beheshti University, Shahid Shahriar Sq., Rashidedin Fazlollah Blvd., Yaman St., Shahid Chamran Hwy., Tehran, Iran.

Tel: +98 (913) 2615259

E-Mail: m_zareei@sbu.ac.ir 


\title{
تأثير غوطدورى ساق و مج يا در آب سرد بر تعادل يوياى ورزشكاران و غيرورزشكاران
}

\author{
روحالله صالحى'، "مصطفى زارعى"، محمدحسين علىزاده'، نورالدين كريمى"
}

ا - كروه طب ورزشي، دانشكده تربيت بدنى و علوم ورزشي، دانشعاه تهران، تهران، ايران.

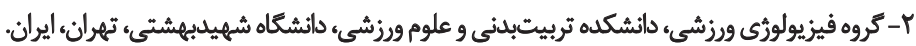

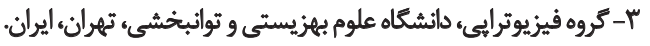

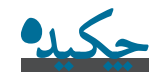

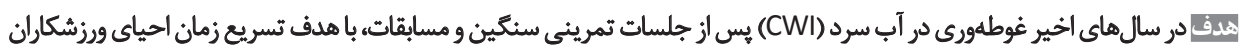

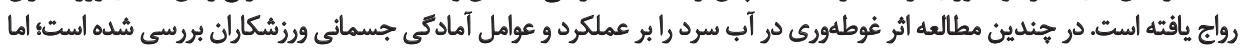

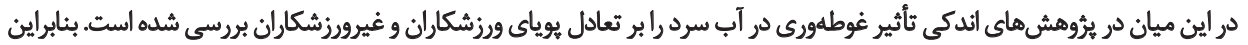

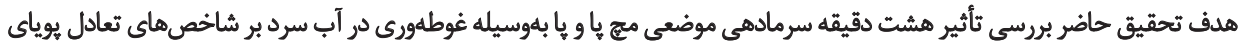

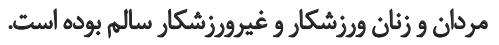

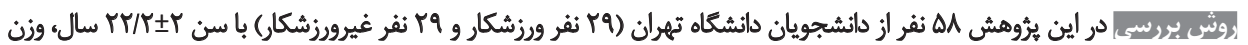

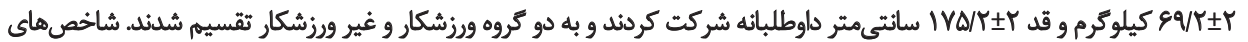

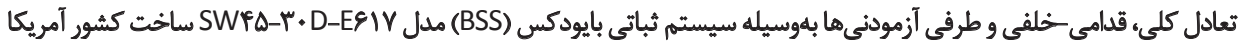

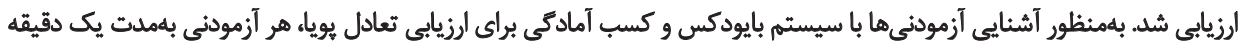

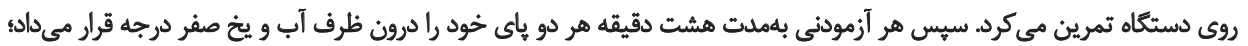

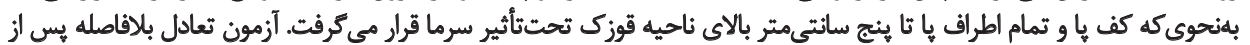

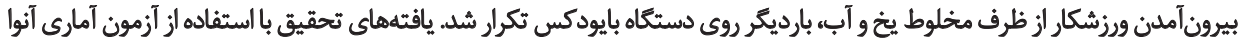

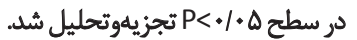

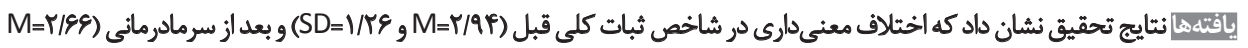

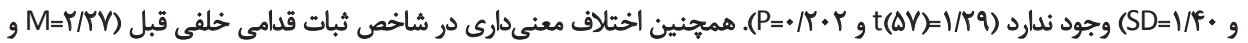

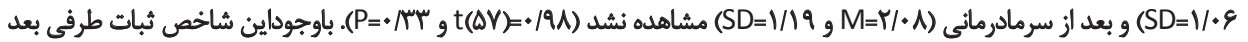

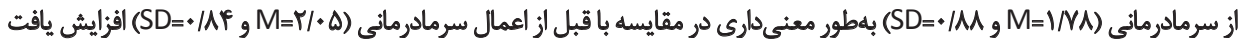

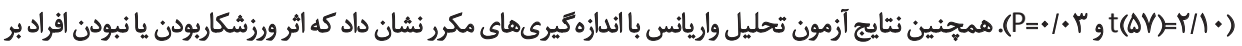

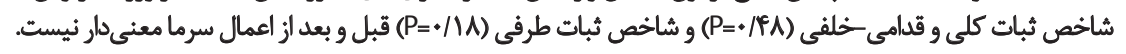

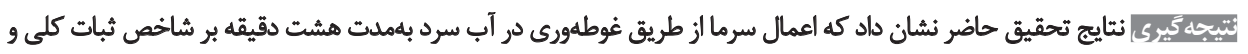

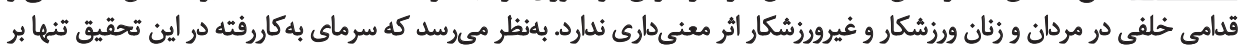

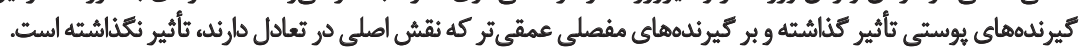

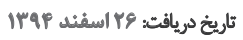

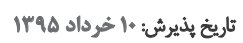

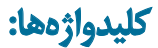
سرمادرمانى، با، ميج يا،

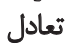

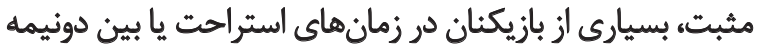

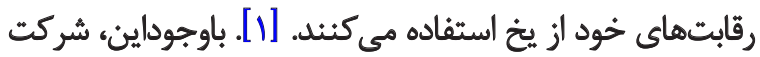

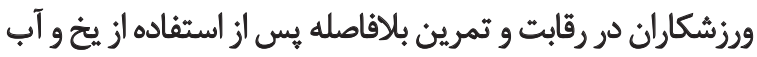

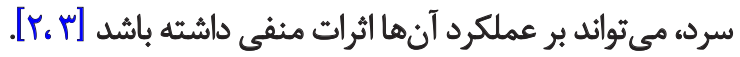

در سالهاى اخير، غوطهورى در آب سرد يس إز جلسات

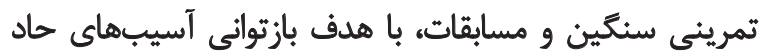

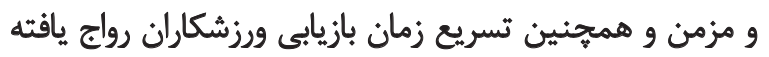

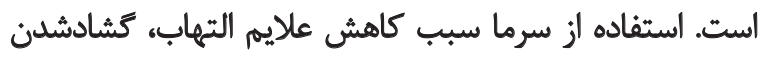

-

دكتر مصطفى زالزعى مئي

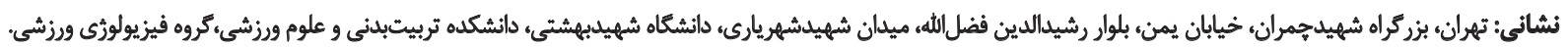
+ m_zareei@sbu.ac.ir رايائامن: 
دانشجويان ورزشكار نخبه تأثير منفى دارد [ب]. علاوهبر تناقض اطلاعات موجود در زمينه تأثير سرما بر تعادل،

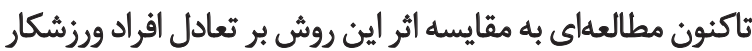

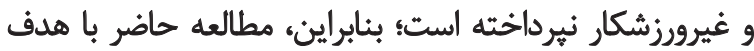

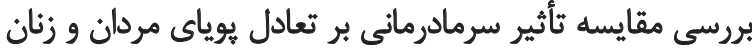
ورزشكار و غيرورزشكار صورت كرفت.

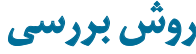

تحقيق حاضر از نوع مطالعات نيمهآزمايشى با طرح بيش آلزمون-

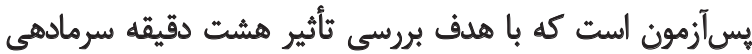

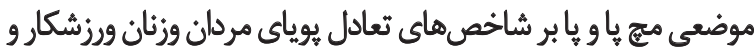
غيرورزشكار سالم شامل تعادل كلى، قدامى خحلفى وطرفى انجام شد.

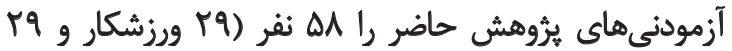
غيرورزشكار) از دانشجويان دانشَّاه تهران (سن

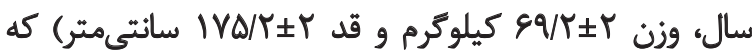

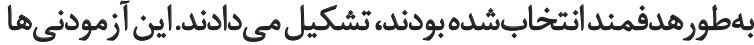

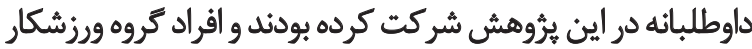

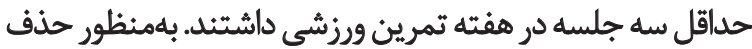

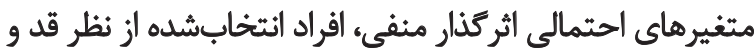

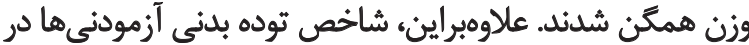

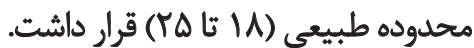

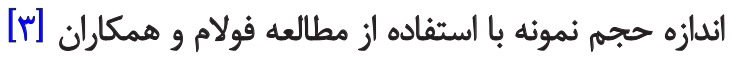

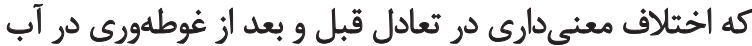

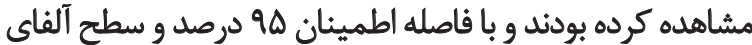

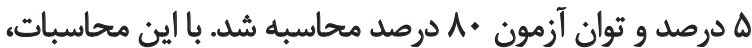

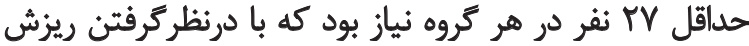

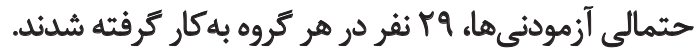
تكميل فرم رضايتثامه و قرارداشتن در محدوده سنى

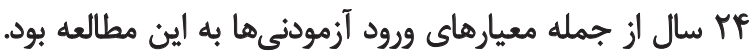

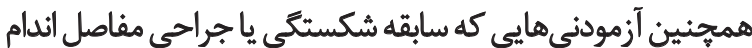

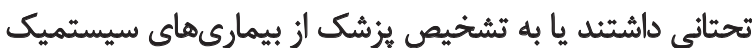

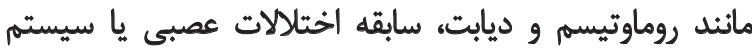

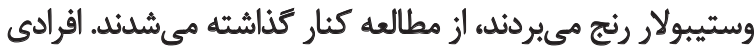

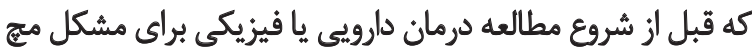

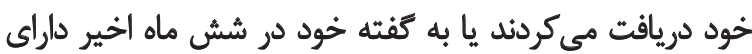

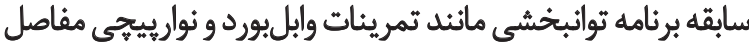

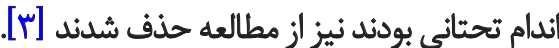

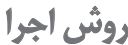

براى ارزيابى تعادل يويا، از سيستم ثباتى بايودكس (BSS)
درهمينراستا، مطالعات بسيارى به بررسى تأثير استفاده از

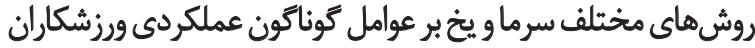

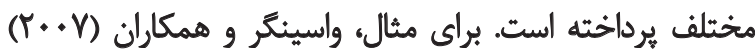

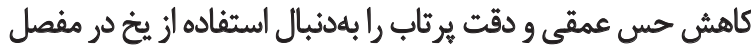

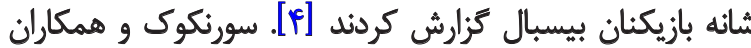

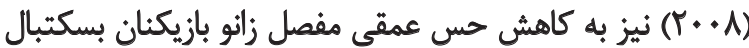

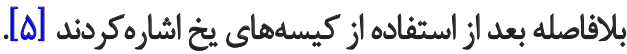

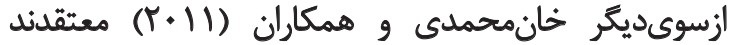

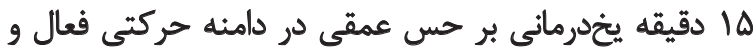

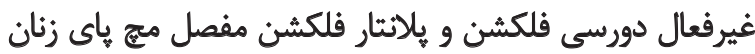

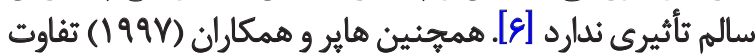

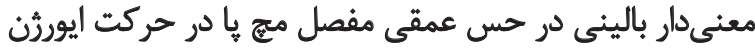

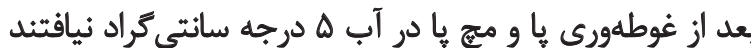

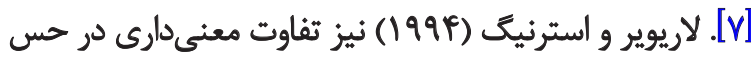

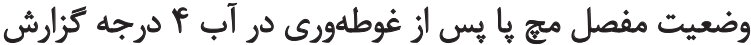

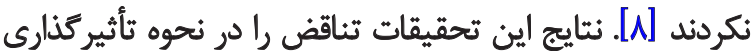

$$
\text { سرما بر حس عمقى نشان مي دهد. }
$$

علاوهبراين، ايوانس و همكاران (1999) نيز نشان دادند كه

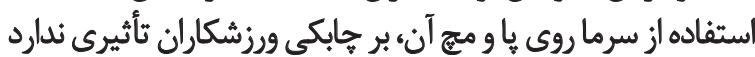

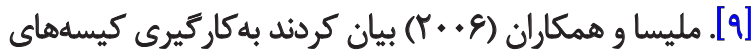

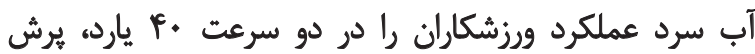

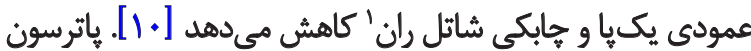

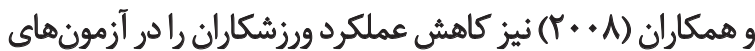

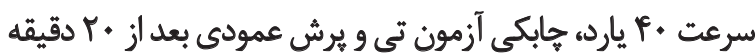

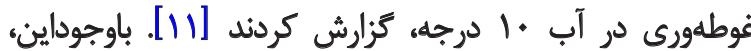

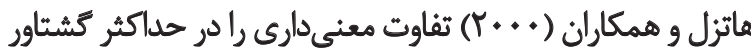

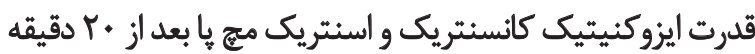

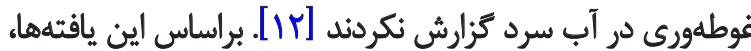

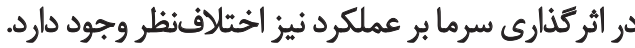
محققان اندكى به بررسى تأثير سرما بر تعادل افراد ورزشكار و

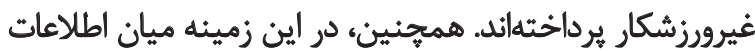

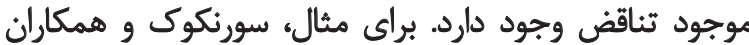

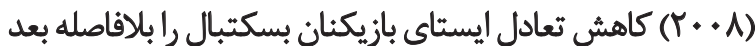

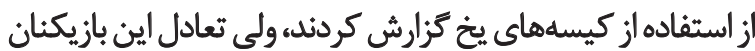

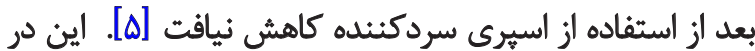

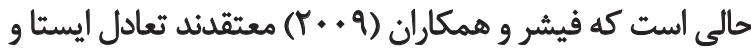

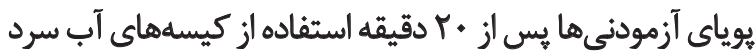

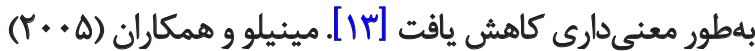

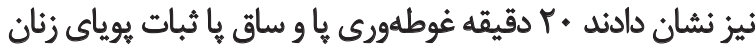

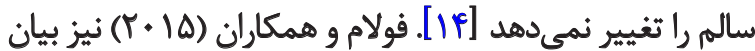

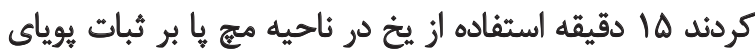


بهمنظور آشنايى آزمودني ها با سيستم بايودكس و ركسب

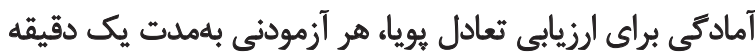

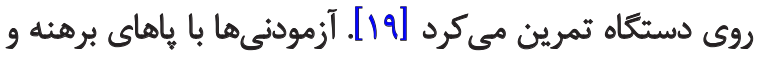

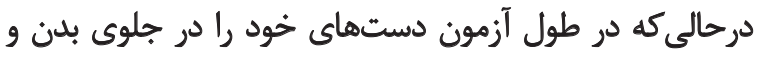

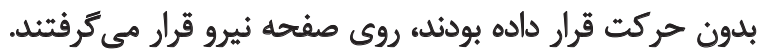

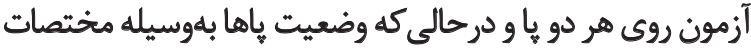

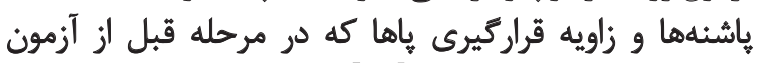

بهدست آمده بوده اجرا مي قشد [19]

آزمودنىها با جشمباز و نكاهكردن به صفحهنمايش سيستم

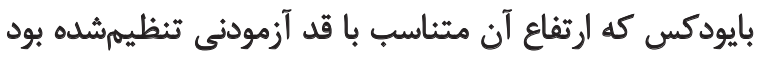

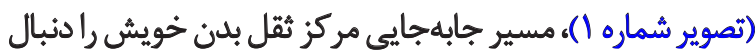

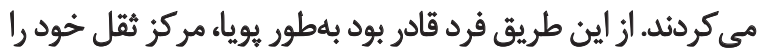

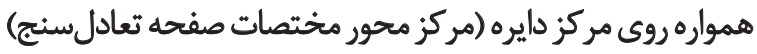

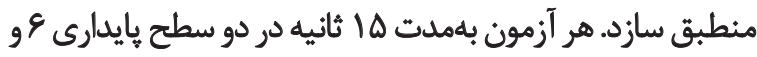

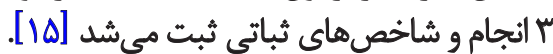

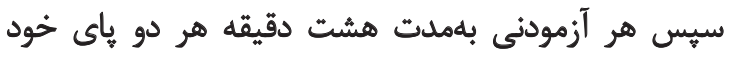

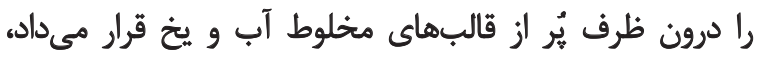

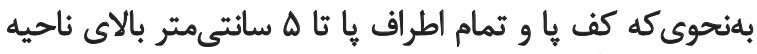

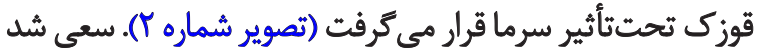

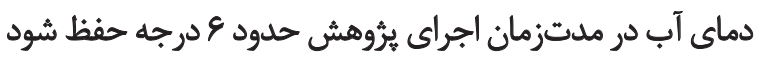

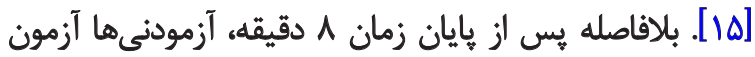

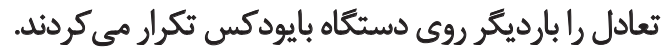

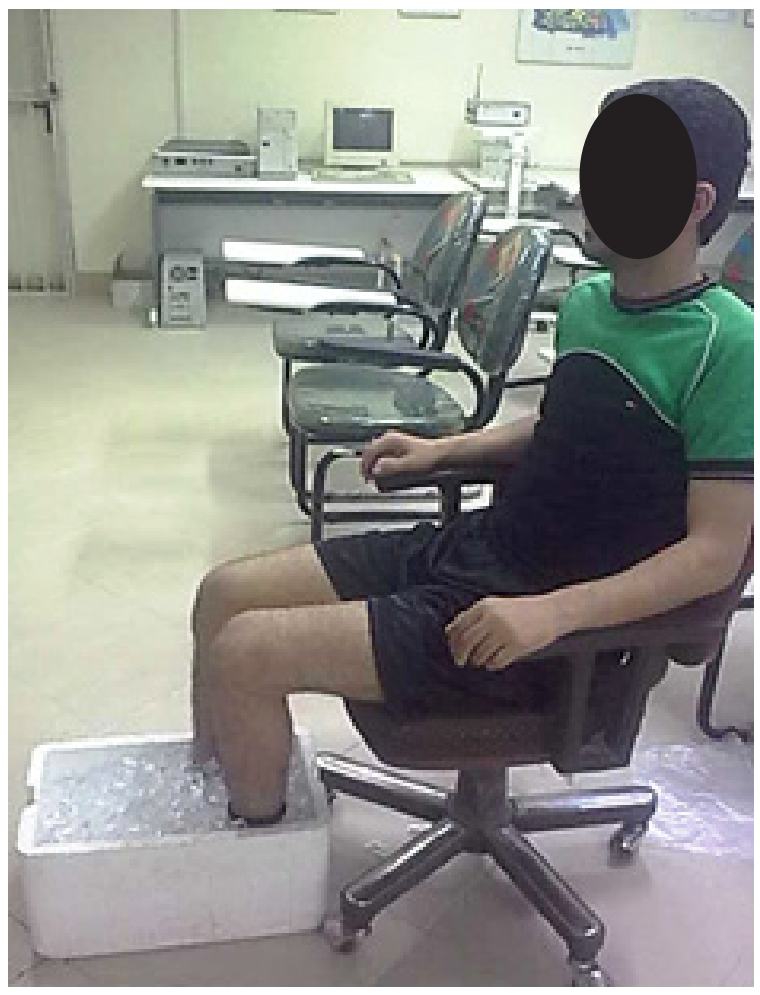

مدل SWFA-r.D-E\&IV ساخت كشور آمريكا استفاده شد.

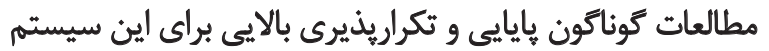

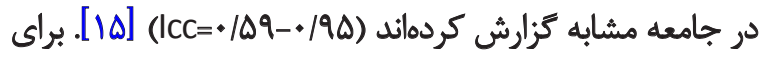

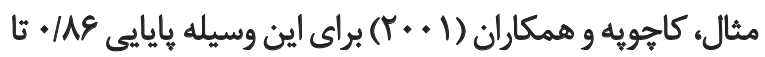

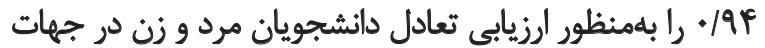

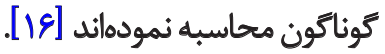

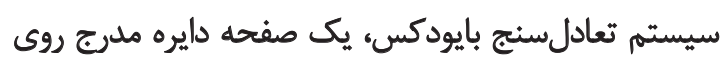

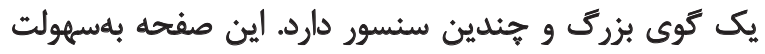

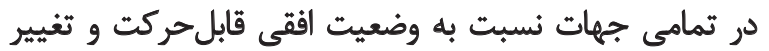

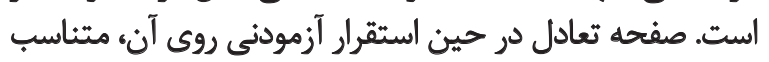

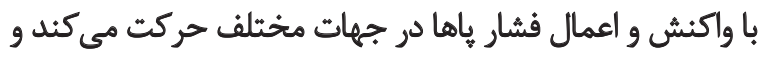

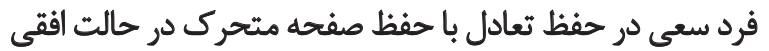

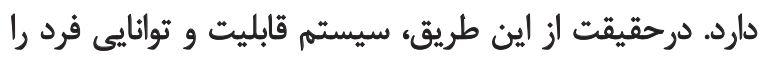

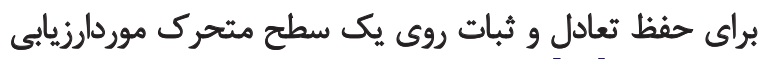

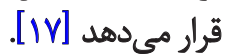

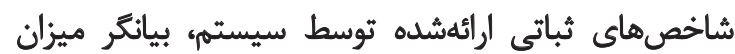

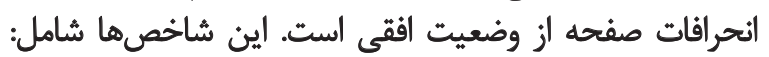

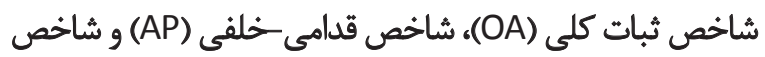

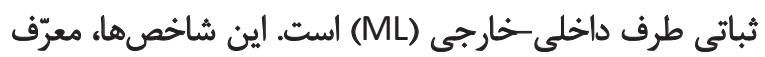

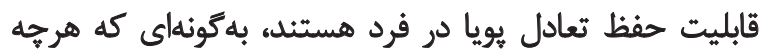

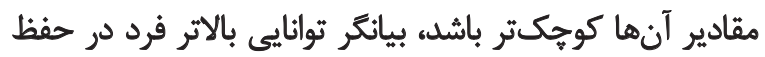

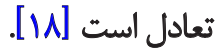

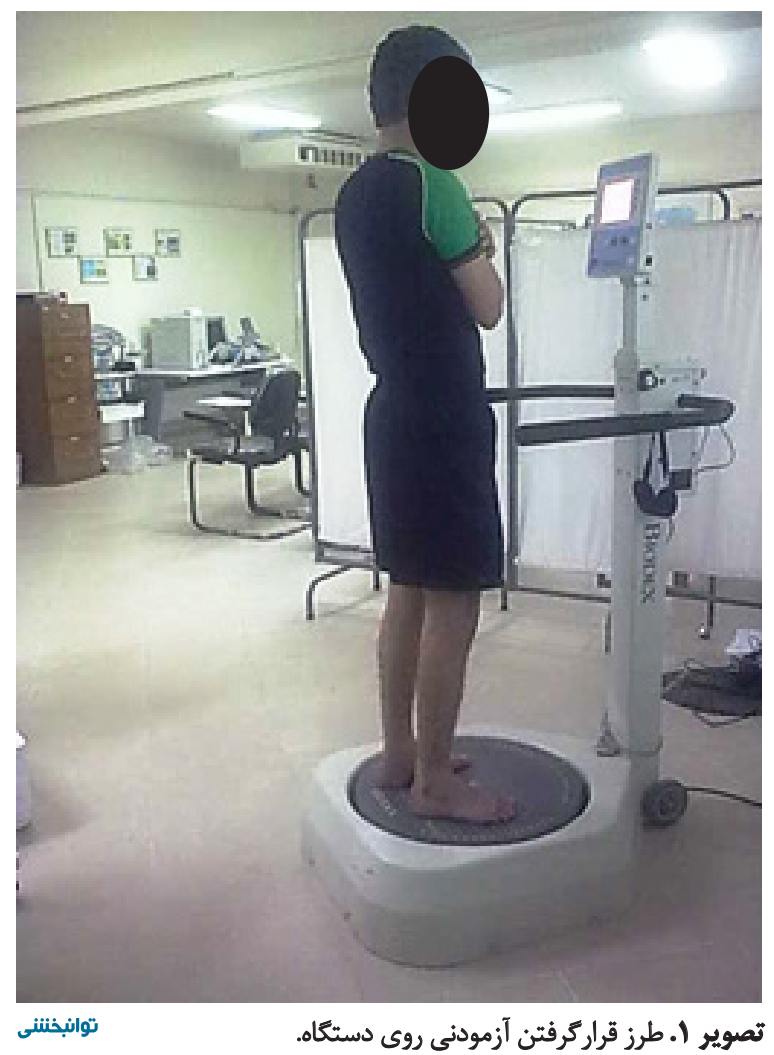




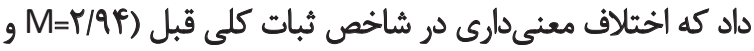

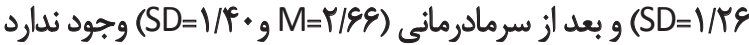
(تصوير شماره $)(P=\cdot / r \cdot r, t(\Delta V)=1 / r q)$

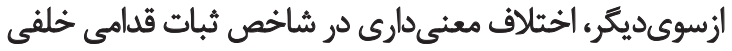

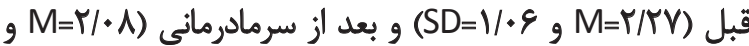
(SD=1/19

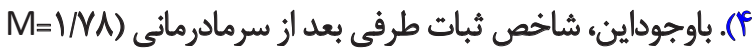

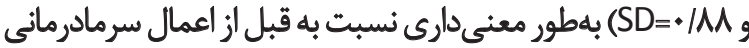

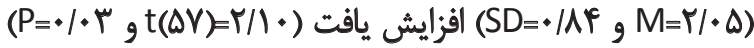

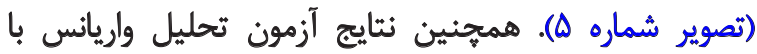

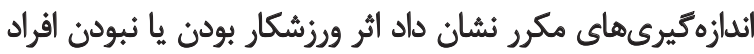

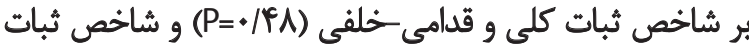
طرفى (P= ) قبل و بعد از اعمال سرما معنى دار نيست.
تمام آزمودنى ها فرم رضايت داوطلبانه و آكاهانه حضور در اين

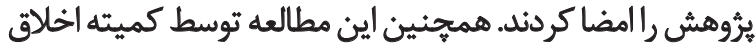

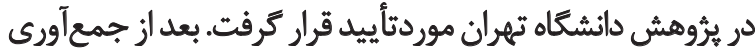

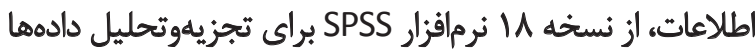

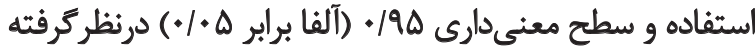

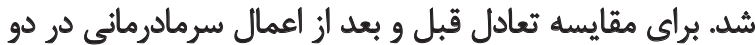

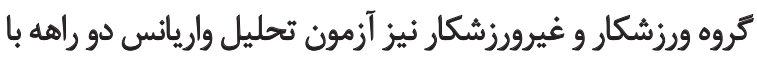

$$
\text { اندازمكيرىهاى مكرر بهكاررفت. }
$$

ياقتهها

مطابق نتايج ارائهشده در جدول شماره ا نشان ميدهد تفاوت

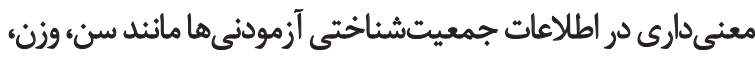
قد و شاخص توده بدنى بين دو كروه وجود ندارد. نتايج تحقيق نشاني

جدول ا. مقايسه متغيرهاى زمينهاى در دو كروه افراد ورزشكار وغيرورزشكار.

\begin{tabular}{|c|c|c|c|}
\hline \multirow{2}{*}{ P-value } & \multicolumn{2}{|c|}{ ميانكين 土|نحراف معيار } & \multirow{2}{*}{ متغير } \\
\hline & غيرورزشكار & ورزشكار & \\
\hline.$/ A T$ & T/GTEYYM & r/UY土Yr/ge & سن (سال) \\
\hline $.1 \Delta \Delta$ & 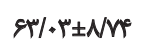 & $8 V / \Delta \Delta \pm 1+/ .1$ & (ونن كيلوكرم) \\
\hline .181 & $199 / \Delta \Delta \pm q / 1 r$ & ISNDADIIITT & (قد سانتى متر) \\
\hline.$/ \mu r$ & $T r / T \cdot \pm 1 / N \Delta$ & $r M / M+1 / r \Delta$ & شاخص توده بدنى (Kg/m \\
\hline
\end{tabular}

$r / \Delta$

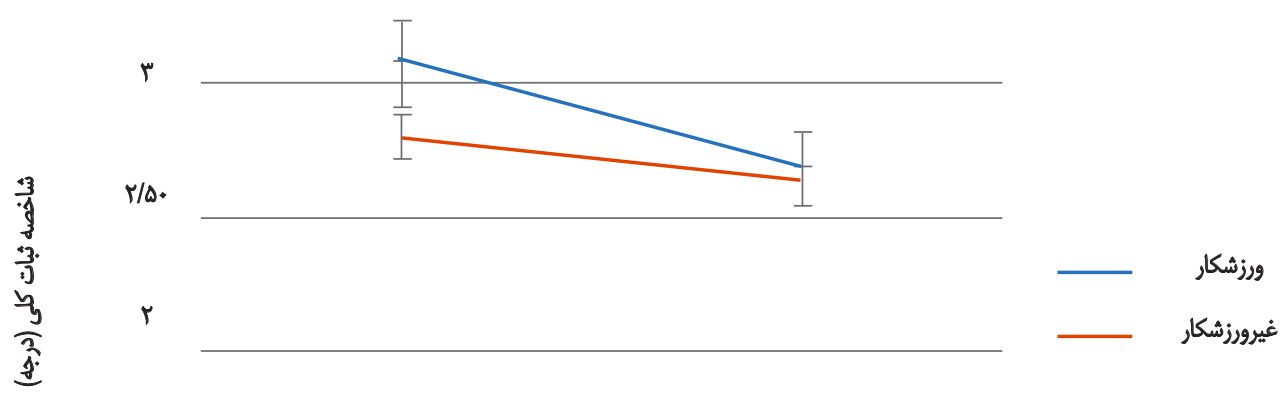

$1 / 4$.

يميشآزمون

بسأزمون 


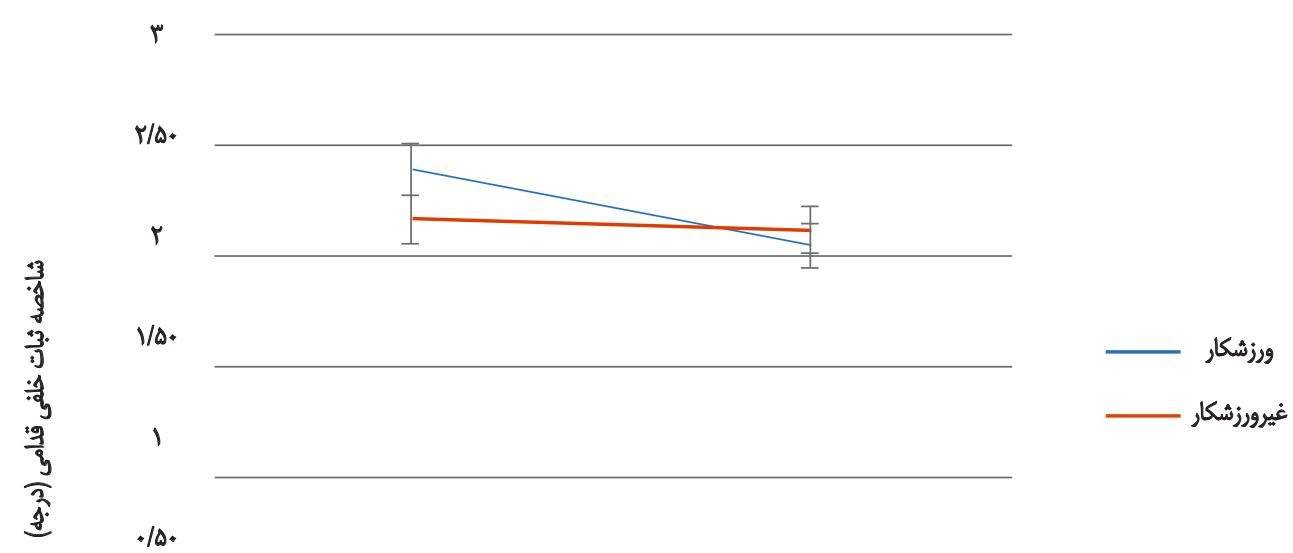

يمأزمون

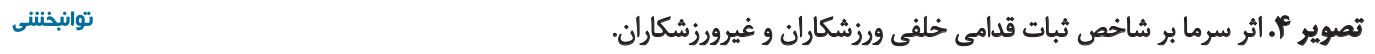

$r$

$r / Q$

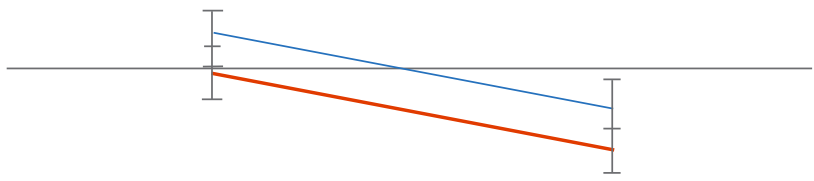

3
$\frac{3}{3}$
$\frac{3}{3}$
$\frac{3}{3}$
$\frac{3}{3}$

$V / \Delta \cdot$

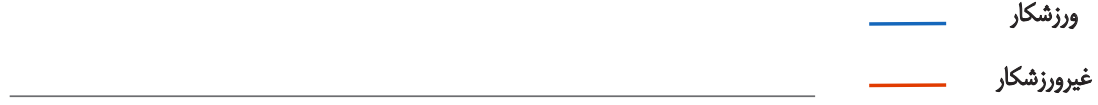

.$/ 4$

ييشن آزهون

بسآزمون

توانبخننى

تصوير هـ اثر سرما بر شاخص ثباتي طرفي ورزشكار ان و غيروزز شكاران.

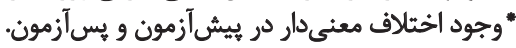

ثبات كلى و قدامى -خلفى و طرفى قبل و بعد از سرمادهى ني

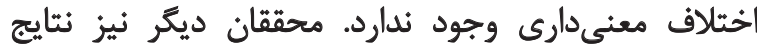

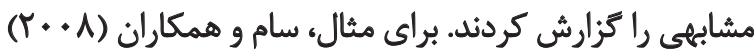

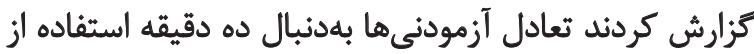

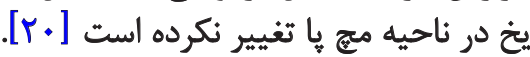

هدف تحقيق حاضر بررسى تأثير هشت دقيقه سرمادهى بـ بادي

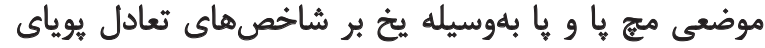

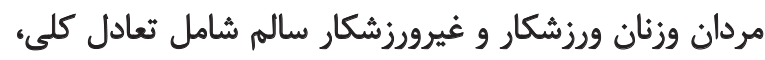
قدامى خلفى و طرفى بود. نتايج تحقيق نشان داد درون در شاخص تمادل 


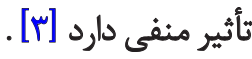

دلايل متعددى براي توجيه تناقض اين نتايج با نتايج مطالعه

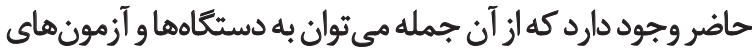

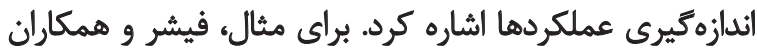

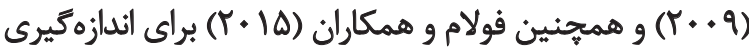

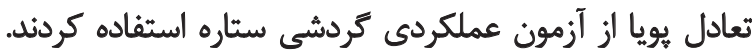

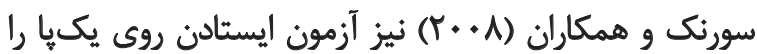

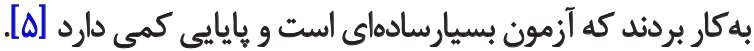

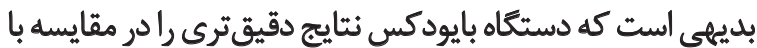

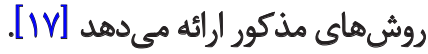

حس عمقى يكى از عوامل مؤثر بر تعادل يويا است. كوستلو و

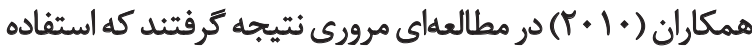

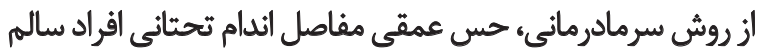

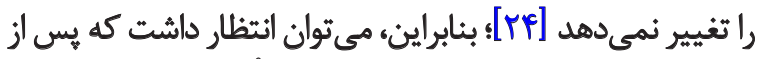
كاربرد سرمادرمانى، تعادل نيز كمتر تحت تأثير قرار تيرد

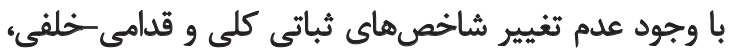

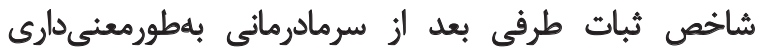

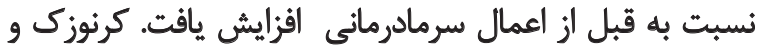

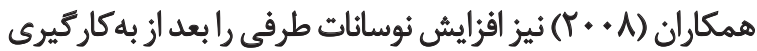

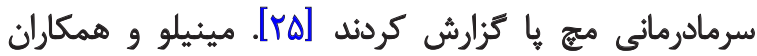

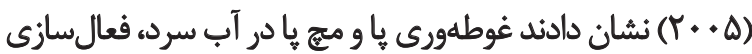

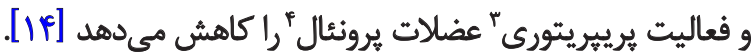

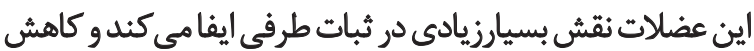

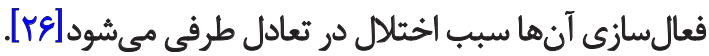

\section{نتيجلكَيرى}

تحقيق حاضر نشان داد كه اعمال سرما از طريق غوطهورى

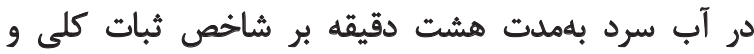

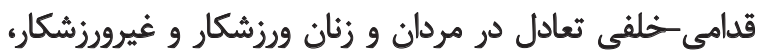

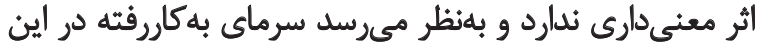

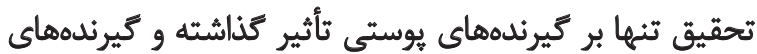

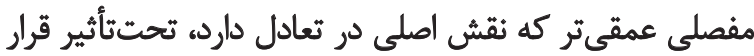

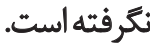

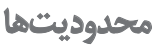

تحقيق حاضر جندين محدوديث احتمالى داشت كه در تفسير

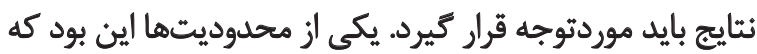

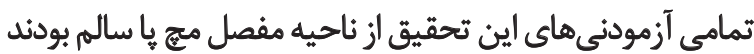

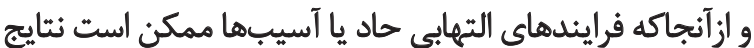

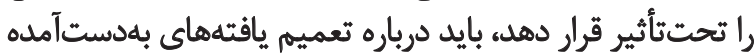

3. Preparatory

4. Peroneal muscles

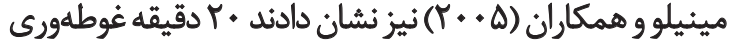

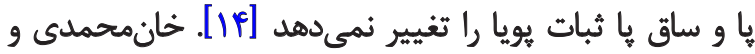

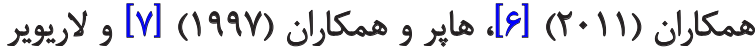

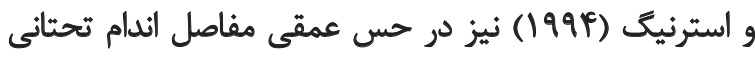

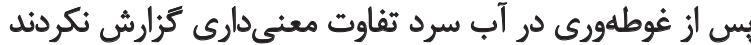

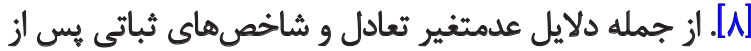

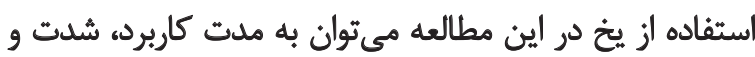
ن استوه استفاده از سرما اشاره كرد.

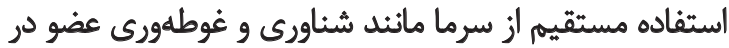

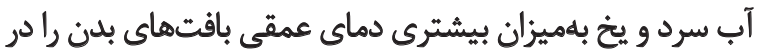

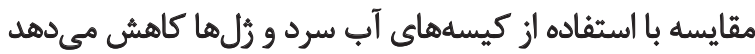

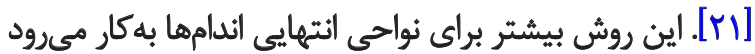

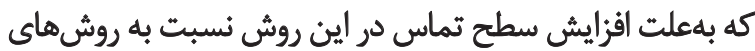

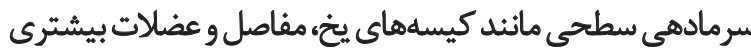
در معرض سرما قرار مي كيرند [عاند].

ازسوى ديكر، مدتزمان استفاده از يخ نيز عامل مهمى بهادمار

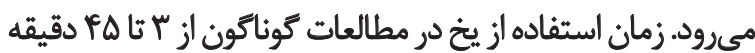

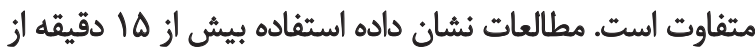

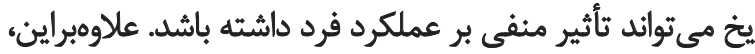

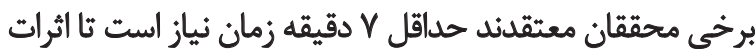

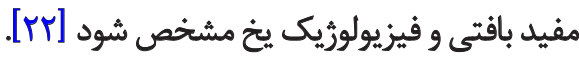

مطالعات ديكرى نشان داده براي دستيابى به اثرات مثبت ديت بإيت

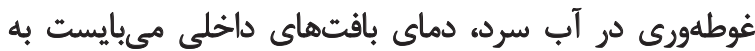

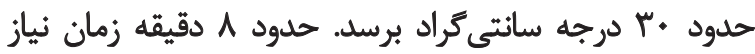

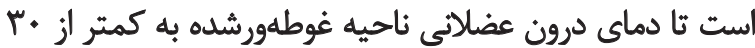

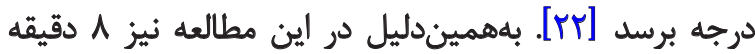

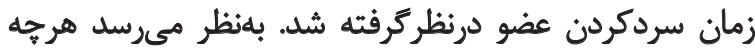

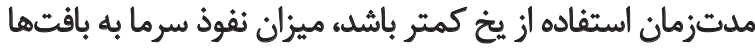

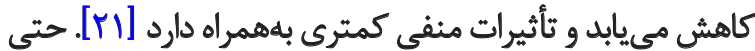

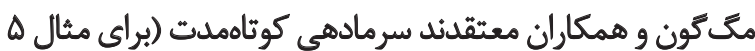

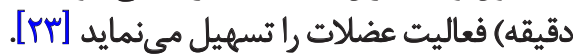

برخى از محققان نيز معتقدئد تعادل و ديكر عوامل عملكردى

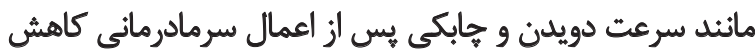

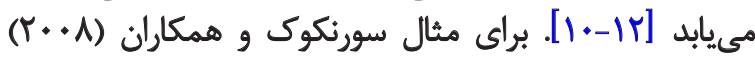

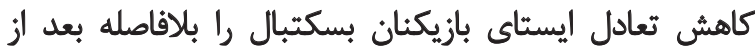

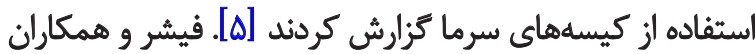

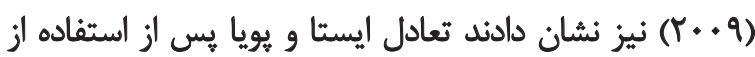

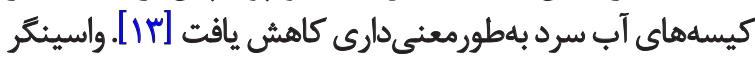

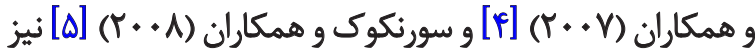

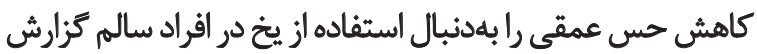

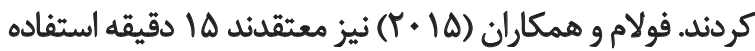

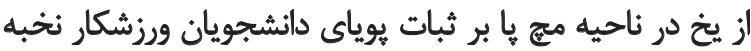




\section{References}

[1] Montgomery RE, Hartley GL, Tyler CJ, Cheung SS. Effect of segmental, localized lower limb cooling on dynamic balance. Medicine \& Science in Sports and Exercise. 2015; 47(1):66-73.

[2] Beyranvand R, Seidi F, Rajabi R, Moradi A. The immediate effect of short-term use of cold spray on ankle joint position sense in healthy individuals. Journal of Research in Rehabilitation Sciences. 2013; 9(5):889-98.

[3] Fullam K, Caulfield B, Coughlan GF, McGroarty M, Delahunt E. Dynamic postural-stability deficits after cryotherapy to the ankle joint. Journal of Athletic Training. 2015; 50(9):893-904.

[4] Wassinger CA, Myers JB, Gatti JM, Conley KM, Lephart SM. Proprioception and throwing accuracy in the dominant shoulder after cryotherapy. Journal of Athletic Training. 2007; 42(1):84-89.

[5] Surenkok O, Aytar A, Tuzun EH, Akman MN. Cryotherapy impairs knee joint position sense and balance. Isokinetics \& Exercise Science. 2008; 16(1):69-73.

[6] Khanmohammadi R, Someh M, Ghafarinejad F. The effect of cryotherapy on the normal ankle joint position sense. Asian Journal of Sports Medicine. 2011; 2(2):91-98.

[7] Hopper D, Whittington D, Davies J. Does ice immersion influence ankle joint position sense? Physiotherapy Research International. 1997; 2(4):223-36.

[8] La Riviere J, Osternig L. The effect of ice immersion on joint position sense. Journal of Sport Rehabilitation. 1994; 3(1):58-67.

[9] Evans TA, Ingersoll C, Knight KL, Worrell T. Agility following the application of cold therapy. Journal of Athletic Training. 1995; 30(3):231-34.

[10] Richendollar ML, Darby LA, Brown TM. Ice bag application, active warm-up, and 3 measures of maximal functional performance. Journal of Athletic Training. 2006; 41(4):364-70.

[11] Patterson SM, Udermann BE, Doberstein ST, Reineke DM. The effects of cold whirlpool on power, speed, agility, and range of motion. Journal of Sports Science \& Medicine. 2008; 7(3):387-94.

[12] Hatzel BM, Kaminski TW. The effects of ice immersion on concentric and eccentric isokinetic muscle performance in the ankle. Isokinetics \& Exercise Science. 2000; 8(2):103-07.

[13] Fisher A. The effect of cryotherapy over the lateral ankle on static and dynamic balance [MSc. thesis]. Pennsylvania: California University; 2009.

[14] Miniello S, Dover G, Powers M, Tillman M, Wikstrom E. Lower leg cold immersion does not impair dynamic stability in healthy women. Journal of Sport Rehabilitation. 2005; 14(3):234.

[15] Schmitz R, Arnold B. Intertester and intratester reliability of a dynamic balance protocol using the Biodex stability system. Journal of Sport Rehabilitation. 1998; 7(2):95-101.

[16] Cachupe WJ, Shifflett B, Kahanov L, Wughalter EH. Reliability of biodex balance system measures. Measurement in Physical Education and Exercise Science. 2001; 5(2):97-108.

[17] Esmaeili E, Salavati M, Maroufi N, Esmaeili V. [Effect of balance board exercises on balance tests and limits of stability by

$$
\begin{aligned}
& \text { از تحقيق حاضر به جمعيتهاي آسيبديده احتياط كرد. }
\end{aligned}
$$

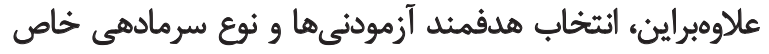

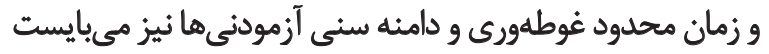

$$
\begin{aligned}
& \text { در تعميم نتايج مدنظر قرار كيرد. } \\
& \text { يشئهاديا }
\end{aligned}
$$

در اين تحقيق تأثير سرمادرماني بر تعادل يوياى ورزشكاران

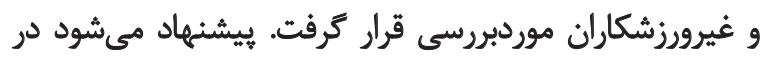

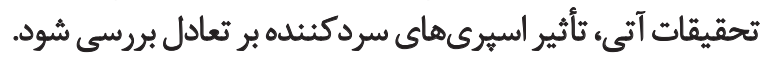

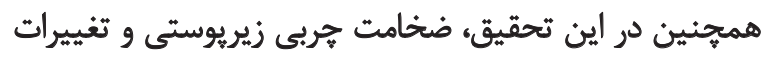

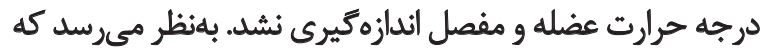
ثبت اين عوامل بتواند به تقويت نتايج تحقيقات آينده كمك كند.

$$
\text { تشكر و قدردائى }
$$

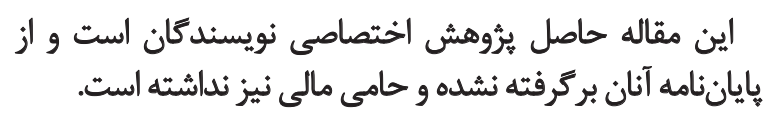


Biodex balance system in normal men (Persian)]. Journal of Rehabilitation. 2006; 7(2):19-25.

[18] Anbarian M, Mokhtari M, Zareie P, Yalfani A. [A comparison of postural control characteristics between subjects with kyphosis and controls (Persian)]. Scientific Journal of Hamadan University of Medical Science. 2010; 16(4):53-60.

[19] Yaghoubi Z, Kahrizi S, Esmaeili E. [The short effects of two spinal stabilization exercise on balance tests and limit of stability in men with non-specific chronic low back pain: randomized clinical trial study (Persian)]. Journal of Rehabilitation. 2012; 13(1):102-13.

[20] Saam F, Leidinger B, Tibesku CO. The influence of cryotherapy of the ankle on static balance. Sportverletz Sportschaden. 2008; 22(1):45-51.

[21] Enwemeka CS, Allen CH, Avila PA, Bina JA, Konrade JA, Munns ST. Soft tissue thermodynamics before, during, and after cold pack therapy. Medicine and Science in Sports and Exercise. 2002; 34(1):45-50

[22] Bleakley CM, Costello JT, Glasgow PD. Should athletes return to sport after applying ice? Sports Medicine. 2012; 42(1):69-87.

[23] McGown HL. Effects of cold application on maximal isometric contraction. Physical therapy. 1967; 47(3):185-92.

[24] Costello JT, Donnelly AE. Cryotherapy and joint position sense in healthy participants: a systematic review. Journal of Athletic Training. 2010; 45(3):306-16.

[25] Kernozek TW, Greany JF, Anderson DR, Van Heel D, Youngdahl RL, Benesh BG, et al. The effect of immersion cryotherapy on medial-lateral postural sway variability in individuals with a lateral ankle sprain. Physiotherapy Research International. 2008; 13(2):107-18

[26] Moghadam M, Salavati M, Ebrahimi E, Arab AM. [The effect of fatigue in proxmal and distal muscles of lower extremity on postural control (Persian)]. Journal of Rehabilitation. 2003; 4(2):2936. 
